\title{
Energetic Disorder Dependence of Optimal Trap Depth in the Space Charge Field Formation for Photorefractivity
}

\author{
Choongkeun Lee, Sun-Kyung Park, Mino Yang, \\ Department of Chemistry, Hanvang Lniversity, Seoul 133-791, Korea. " E-mail: kimnjahamang.ac.kr \\ ${ }^{\dagger}$ Institute of Sciences and Technologv: College of Science and Technolog, Korea Lniversity, Chungham 339-700, Korea \\ -Department of Chemistry, Chungbut National University, Cheongin 361-763, Korea \\ ${ }^{\circ}$ E-mail: minovangachungbuk.ackr; nslee achungbuk.ackr \\ Received December 16, 2006
}

\begin{abstract}
Trap effects on the formation of space-charge field $(\mathrm{SCF})$ associated with the photorefractivity of nonlinear optical polymers were studied by the Monte Carlo simulation using modified Gaussian disorder model. The charge transport dy'namics influenced by the presence of trap molecules controls the formation of SCF via the charge distribution. Temporal behavior of SCF formation and SCF dependence on the trap depth are discussed in terms of the concentration and distribution of charges (holes and ionized acceptors) developed following illumination of light. The correlation of the trap depth and the energetic disorder is presented for an optimal efficiency for the SCF formation.
\end{abstract}

Key Words : Photorefractivity, Charge transport dynamics. Space-charge field. Disorder and trap depth Monte Carlo simulation

\section{Introduction}

Optical devices faster more sensitive and more reliable. have been intensively developed to replace electronic devices for many y'ears. Therefore. materials of which optical properties are sensitive to light illumination. including nonlinear optical (NLO) materials. have attracted much attention. ${ }^{1.2}$ One of properties of NLO materials is a photorefractive (PR) effect that is attributed to the spatial modulation of the index of refraction under non-uniform light illumination. This electro-optic non-linear effect involves the space charge field (SCF) formation. The SCF arises when charge carriers photo-generated by spatially modulated light excitation are separated by drift and/or diffusion processes and accordingly' become trapped to produce a non-uniform charge distribution. ${ }^{1-3}$

The characterization of SCF in PR polymers had been performed on the variation of photorefractivity with the SCF. ${ }^{3-1}$ The magnitude of SCF was determined experimentally by observing electro-optic coefficients measured with a Mach-Zelunder interferometer. ${ }^{16}$ This method had been frequently employed to determine the $\mathrm{SCF}^{11.12}$ However. it was rather not a routine work to obtain effective electro-optic coefficients appropriately in various kinds of material systems and experimental conditions. Therefore. other optical methods were proposed for the magnitude of SCF in polymeric PR materials. In these methods. the SCF was determined from a modified four-wave mixing technique using polarizers or from birefringence measurement method. ${ }^{8.9}$ These experimental results showed a linear dependence of SCF on the external applied field. and a linear dependence of the diffraction efficiency on the absolute extent of SCF. ${ }^{8-1]}$

The SCF formation processes have been theoretically studied by solving kinetic differential equations for the time- and space-dependent densities of holes. ionized acceptors. and traps to predict PR dynamics from basic material properties. ${ }^{3-7}$ These theoretical studies have been rewarding in reproducing the SCF but have limitations in understanding the formation dynamics of SCF in terms of molecular properties of PR materials. Another approach utilizes the method of Monte Carlo (MC) simulation. MC simulation method has been adapted to elucidate charge carrier transport and/or charge redistribution appearing in various devices. semiconductor. conjugated polymer. molecularly doped polymer. etc. by incorporating readily the molecular properties of charge transport materials and trap molecules into the sy'stem. ${ }^{13-23}$ In those works. MC simulation has successfully described the charge carrier transport and/or redistribution dynamics in the system including molecular parameters.

In the present work, we have examined the effects of doped traps on the formation of SCF in the photorefractive film system using MC simulation. In the model adapted. the doped traps are characterized simply by shifting their mean energy from that of the hole transport sites (HTSs) by a certain extent called a trap depth. In such a system. it may be functional to predict the PR dynamics directly from a set of basic material properties in order to lead the development of new materials. Based on a series of MC simulations. we shall reveal a quantitative relation between the trap depth and the energetic disorder of HTSs to exhibit an optimal efficiency in the formation of SCF. In addition. we also present details of the distribution development of charges (holes and ionized acceptors) responsible for the formation of SCF with the temporal scope and with the trap depth.

\section{Simulation}

In this work. the Gaussian disorder model (GDM) developed 
for a theoretical study of photoconductivity was employed with some modifications. ${ }^{19,2(1} \mathrm{MC}$ simulations were performed on a cubic lattice of $70 \times 50 \times 50$ sites $(x \times y \times z)$ with a lattice constant $a=10 \AA$ that is typical for most organic matrices. ${ }^{15,2 i}$ The periodic boundary condition was introduced along $y$ and $z$ axes. The lattice sites were randomly assigned as either an HTS (70\%) or a doped trap site $(30 \%)$. The site energies were assumed to obey a Gaussian distribution function with a standard deviation $(\sigma)$ that characterizes the degree of energetic disorder of HTSs. Ten configurations of the site energies were sampled to get meaningful statistics. Spatially uniform electric field. $E_{u}$. was applied along the $x$-direction through the lattice sites confined by two electrodes at both sides (i.t. an anode at $x=0$ and a cathode at $x=x_{\text {(nax }}$ ).

Pairs of an ionized acceptor (with a negative charge) and a hole (with a positive charge) are created at vacant HTSs by illumination of light of which the intensity is sinusoidally modulated across the $x$-direction. The applied electric field drives the holes moving toward the cathode while the ionized acceptors are assumed immobile. Once a hole/ ionized-acceptor pair is created. the hole can hop to a neighboring (not necessarily nearest) HTS if not occupied by another hole. The hopping rate was assumed to follow the Miller-Abrahams theory. sites was adjusted by Coulomb interaction energies with ionized acceptors located within the Coulomb radius. As a hole encounters an ionized acceptor. they were set to vanish immediately (electron-hole recombination). Arriving at the cathode a hole is removed from the system and another hole is created at the anode to balance the total charge of the system. Other simulation conditions may be referred to our previous works. $^{2 \geq}$

SCF formed was obtained by solving the Poisson's equation:

$$
\frac{\partial E}{\partial x}=\rho(x)+N^{-}(x)
$$

with the boundary condition

$$
\frac{1}{x_{\max }} \int_{0}^{x_{\max }} E(x) d x=E_{a}
$$

In equation (1). $\rho(x)$ and $N^{-}(x)$ denote the charge densities of the holes and ionized acceptors. respectively at a location $x$. The generated SCF implicates the distribution of charges by shifting the site energies and consequently changing the hopping rates and/or by altering the yield of charge generation (as a charge generation rate depends on the electric field). The charge distribution again determines the profile of the evolved SCF, and consequently SCF was calculated self-consistently in the course of our simulation. We introduce the root mean square (RMS) of SCF as a measure of magnitude of the generated SCF as following:

$$
\sigma_{E}=\sqrt{\frac{1}{x_{\max }} \int_{0}^{x_{\mathrm{rtr}}}\left(E(x)-E_{u}\right)^{2} d x}
$$

\section{Results and Discussion}

In this study. we have explored the SCF formation dynamics upon variation of the trap depth that is one of the control variables for the SCF formation. The shapes of SCFs at the steady states obtained through MC simulations were similar in shape to Schildkraut's or Yuan's obtained solving the rate equations. ${ }^{3.7}$ The amplitude and phase of SCF are dependent on the trap depth because the distributions of trapped holes and ionized acceptors differ to the trap depth. Temporal evolutions of the hole concentrations (the number concentration of holes. here) and the RMS of SCF are presented in Figure 1. This illustrates that two quantities. the hole number and SCF, are strongly relevant to the trap depth. In Figure 2, we have shown the spatial distributions of the holes and ionized acceptors. for a trap depth of $0.4 \mathrm{eV}$. spread along the $x$ axis at three specific instants in the course of temporal development. The distributions of the holes and ionized acceptors are distinct apparently at initial early stage $(0.2 \mu \mathrm{s})$. the maximal point $(2.5 \mu \mathrm{s})$, and the steady state $(15.0 \mu \mathrm{s})$ to generate the holes and produce the SCF. These

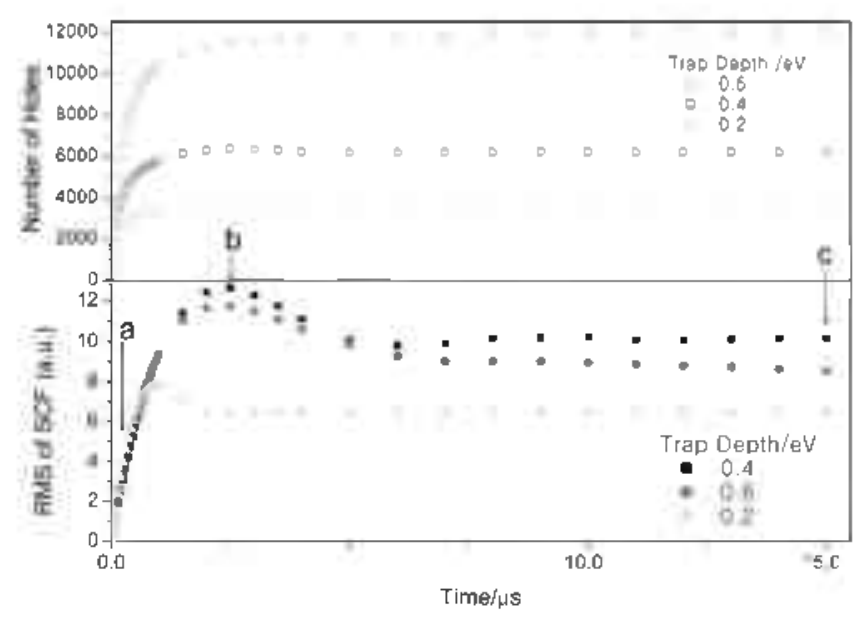

Figure 1. Time dependence of the RMS of SCF (Bottom), and the hole concentration (Top). $\left(E_{q}=80 \mathrm{~V} / \mathrm{m} \mathrm{m}\right.$ and $\sigma=0.05 \mathrm{eV}$ )

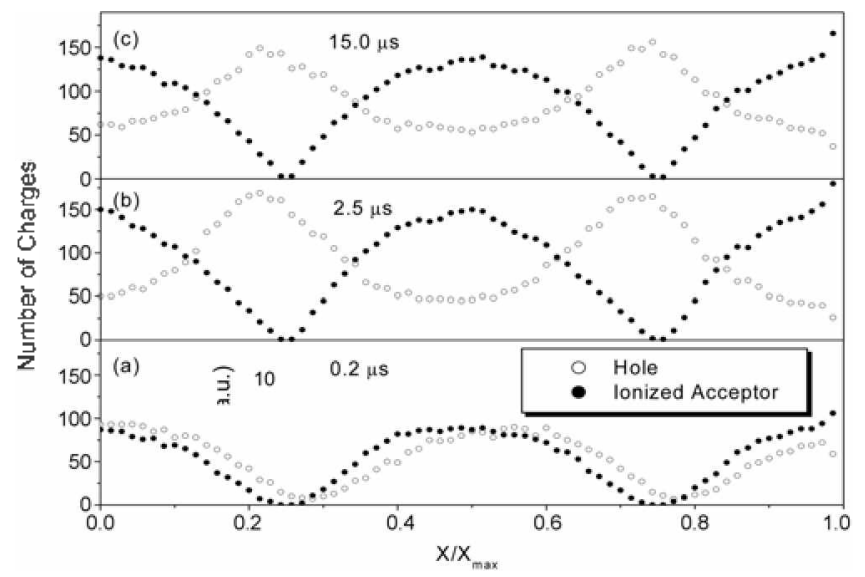

Figure 2. The evolution of the holes and the ionized acceptors at (a) $0.2 \mu / \mathrm{s}$ (b) $2.5 / l \mathrm{~s}$ and (c) $150 \mu / \mathrm{s}$. ( $E_{\sigma}=80 \mathrm{~V} / \mu \mathrm{m}, \sigma=0.05 \mathrm{eV}$, and Trap depth $=0.4 \mathrm{eV}$ ) 
three points are indicated using arrows on Figure 1.

Figure 2(a) shows that the holes generated by excitation launch to separate from their geminate partners and to drift toward the cathode responding an applied electric field. This process for the charge separation should occur prior to the development of SCF. When the hole concentration becomes raised adequately at $0.4 \mathrm{eV}$ of the trap depth in this case and the charges are effectively distributed as display'ed in Figure 2(b) corresponding to the maximal point b (at $2.5 \mu \mathrm{s}$ ) in Figure 1, the amplitude of SCF exhibits its maximum. The peak position in the distribution curve of the immobile ionized acceptors is a little shifted to the anode (at $x=0$ ) with weak asymmetric profile. This reflects the creation of the ionized acceptors by the induced SCF and also spatially inhomogeneous depletion of concentration resulting from the charge recombination process. At the steady state. around the point $c$ in Figure 1. the hole concentration is slightly smaller than that at maximal point $b$ because the rate of charge recombination process has increased at the steady state. The intensity of SCF at the steady state. however. is significantly diminished as the concentration of net charges becomes reduced. The reduction of net charges results mainly' from the charge recombination and/or the neutralization of holes and ionized acceptors. which are taking place as mobile holes reach a region of ionized-acceptor-rich. After $5.0 \mu \mathrm{s}$. the RMS of SCF shows weak oscillatory behaviors. The appearance of such phenomenon is consequent from contiguously occurring two processes. the charge separation and neutralization.

In Figure 3. we present the dependence of the RMS of SCF and of the hole concentration at the steady state on the trap depth. The hole concentration at the steady state increases monotonically with the trap depth because the rate of recombination process is reduced by retarded mobility of holes. The intensity of SCF at the steady state. however. shows a tumover behavior with respect to the trap depth. This phenomenon can be explained in terms of the charge distributions varying with changing trap depth. Figure 4 shows spatial distributions of holes and ionized acceptors attained at the steady state for three different trap depths. 0.2 .

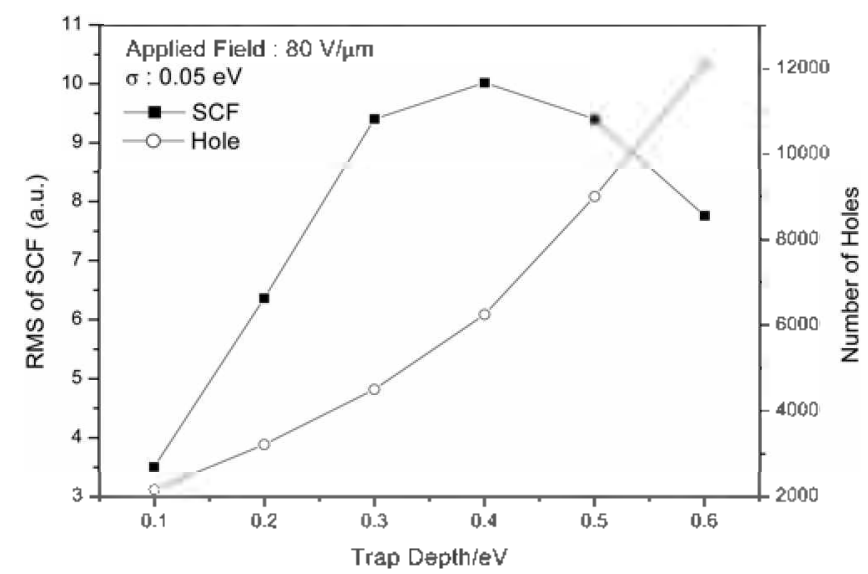

Figure 3. RMS of SCF and the hole concentration to the trap depth. $\left(E_{\sigma}=80 \mathrm{~V} / \mathrm{mm}\right.$ and $\left.\sigma=0.05 \mathrm{eV}\right)$

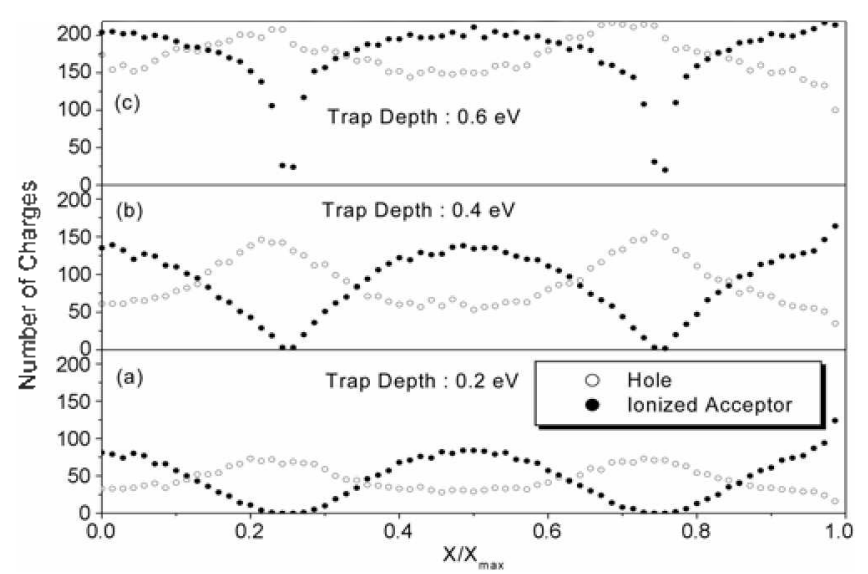

Figure 4 . The distribution of the holes and the ionized acceptors at the steady state for the trap depth of (a) $0.2 \mathrm{eV}$, (b) $0.4 \mathrm{eV}$ and (c) $0.6 \mathrm{eV}$ at $E_{\sigma}=80 \mathrm{~V} / \mu \mathrm{m}$ and $\sigma=0.05 \mathrm{eV}$.

0.4 and $0.6 \mathrm{eV}$. respectively: As the trap depth gets small. mobile holes separated from their geminate ionized acceptors move on easily toward the cathode. In this case. SCF grows fast at early stage as shown in Figure 3. but its intensity at the steady' state is not well-built yet because of less charge concentrations by promoted recombination process. as shown in Figure 1. In the case of large trap depth. the migration of holes is considerably retarded due to large trap depth. Consequently most holes settle down at neighboring sites of their geminate ionized acceptors. Then the distribution curve of holes overlaps mostly with that of ionized acceptors as shown in Figure 4(c). In this case the intensity of SCF tuns diminutive by simple neutralization of the charge aggregation in spite of large hole concentration. When the trap depth is adjusted to be adequate. holes and ionized acceptors are effectively distributed for SCF formation and then the intensity of SCF gains its maximal value compared to other cases. As discussed above. the charge concentration and distribution is subject to the extent of the trap depth. As a result. the strength of generated SCF determined by the charge concentration and distribution is strongly dependent on the trap depth. So we may expect that there should be an optimal value of the trap depth coupled with other variables of the system such as the energetic disorder. an applied field and so on.

In order to explore the interplay between the energetic disorder and the trap depth. we draw three dimensional plot for RMS values of SCF at the steady state as a function of the standard deviation of the site energy distribution. $\sigma$. and the trap depth in Figure 5 (Right). We can see that. for a given energetic disorder, the intensity of SCF shows an oscillatory behavior spanning the axis of trap depth. However. the intensity turns over downward after the trap depth reaches a certain value. We could call it an optimal trap depth for a given energetic disorder. For the domain of the trap depth larger than this optimal depth. the intensity of SCF decreases with the trap depth. The decreasing appears because the rate of the charge separation process accordingly decreases down when the trap depth is far beyond the optimum. When the 

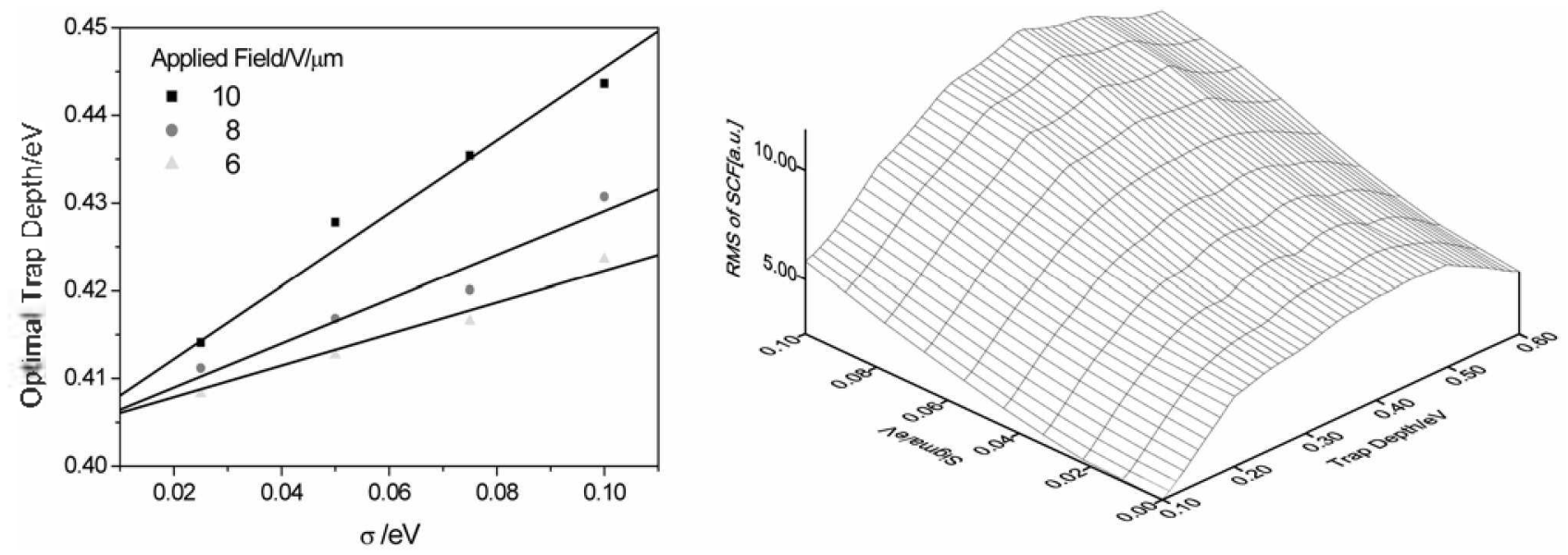

Figure 5. Optinal trap depth as a function of the energetic disorder (Left), and 3-D plot for RMS of SCF at the steady state as a function of $\sigma$ and the trap depth, $E_{n}=60 \mathrm{~V} / \mathrm{tam}$. (Right)

trap depth is smaller than the optimal depth. the intensity of SCF does not gain its intensity by facile recombination process due to elevated mobility of holes. In other words. the optimal trap depth corresponds to the depth yielding an effective charge distribution. as shown in Figure 4(b). minimizing the loss of charges due to the hole/ionized acceptor recombination and/or charge neutralization.

While the trap depth is a characteristic feature of electronic structure of the doped trap molecules. the energetic disorder is determined by the molecular interactions mainly between the charge carrier molecules. partly between doped materials. and partly between the charge carrier molecules and doped materials. The interactions in total are also exercised by concentrations of components ${ }^{21.20,35}$ We present the optimal trap depth as a function of the energetic disorder in Figure 5 (Left). We can observe a clear correlation between two variables. As the energetic disorder of HTSs gets larger the trap depth should be increased in order to retain the trapped holes from transferring to nearby HTSs and to manage to gain a large intensity of SCF. This observation suggests that we may control the trap depth to improve the efficiency of SCF fomation for a given sy'stem with an energetic disorder. The account of the optimal trap depth on the energetic disorder is requisite to lead the development of new materials and/or to select NLO materials with maximal efficiency of SCF for a șistem.

\section{Conclusion}

The present work illustrates that $\mathrm{MC}$ simulation method may well describe the formation dynamics of SCF in PR polymer system. This study shows that the strength of generated SCF determined by the charge concentration and distribution is strongly dependent on the trap depth. To obtain a maximal efficiency of SCF intensity. one should make a choice of a trap material with an optimal trap depth that is correlated with the energetic disorder of charge carrier molecules and/or the applied field.
Acknowledgement. We acknowledge the Korea Science and Engineering Foundation for the financial support.

\section{References}

1. Moerner. W. E.: Silence. S. M. Chem. Rev: 1994. 94. 127.

2. Ostroverkhova. O.: Moerner. W. E. Chem. Rev: 2004. 104. 3267.

3. Ostroverkhova. O.: Singer. K. D. J. Appl. Phys. 2002.92.1727.

4. Schildkraut. T. S.: Buettner. A. V. J. Appl. Phis 1992. 72.1888

5. Schildkraut, J. S.; Cui, Y. J. Appl Phys. 1992. 72, 5055

6. Yuan. B.: Sun, X; Hou, C; Li. Y: Zhou, Z; Jiang. Y; Li, C. d. Appl. Plo's. 2000, 88.5562

7. Yuan. B.: Sun1. X: Zhou. Z: Li. Y.: Tiang. Y.: Hou. C. J. Appl. Pho 2001. 89.5881

8. Joo. W.-T: Kim. N.-T.: Kim. H. C.: Moon. I. K.: Kim. N.: Oh. C.H. J.Appl. Phys 2002. 91,6471

9. Hwang. U.-J, Choi. C.-S.: Vuong, N. Q.: Kim, N. J. Chem Plys. 2005. 123,244905

10. Moerner. W. E.: Silence. S. M.: Hache. F.: Bjorklund. G. C. J. Opt. Soc. Ant B 1994. H. 320 .

11. Swedek. B.: Cheng. N.: Cui. Y.: Zieba. T.: Winiarz. T.: Prasad. P. N. J. Appl. Plys. 1997.82,5923.

12. Jakob. T.: Schloter. S.; Hofmann, U.: Grasruck. M.: Schreiber, A.: Haarer. K. J. Chem. Phys. 1999, 111, 10633.

13. Fischetti. M. V: Laus. S. E. Phus Rev: $B$ 1988. 38.9721.

14. Lee. C. T. Bull. Korean Chem. Soc. 2006. 27, 1186.

15. Farouki. R. 1: Hamaguchi, S.: Dalvie. M. Plys. Rev \& 1991, 4. 2664.

16. Simmerer. T. J.; Barnes. M. S.: Keller, J. H.; McCaughey. M. J.; Kushner. M. J. Appl. Phys. Lett. 1991. 59,638.

17. Feller. F.: Geschke. D.: Monkmant. A. P. Appl. Phs. Lett 2001. 79.779 .

18. Upadhyaya. G. S.: Shohet. T. L.: Lauer. T. L. Appl. Phys. Lett. 2005. 86,1021

19. Schonherr. G.: Bassler. H; Silver, M. Phil. Mag. B 1981. H4, 47

20. Novikov, S. V: Vannikov. A. V. Synth. Het. 1997,85. 1167.

21. Nespurek. S.: Sworakowski. J. Thin Solid Films 2001. 393. 168.

22. (a) Lee. C.: Yang. M.: Lee. N.-S: Kiml. N. Chem. Phns Lett. 2006. H18. 54 (b) Lee. C.: Park. S.-K.: Yang. M.: Lee. N.-S.: Kim. N. Chem Plys. Lett. 2006. +22, 106.

23. Han. S. W. Jang, J. Bull. Korean Chem. Soc. 2006, 27,31

24. Miller. A.: Abrahams. E. Phys. Rev 1960. 120,745.

25. Bassler. H. Phys. Status Solidi B 1993. 175. 15. 